\title{
Superior Retinacular Artery Did Not Occlude in a Rat Model of the Non-Traumatic Osteonecrosis of the Femoral Head
}

\author{
Shunichiro Okazaki1,2, Satoshi Nagoya1, Junya Shimizu',3, Nobuyuki Takahashi2,3, \\ Keisuke Mizuo², Hideki Hyodoh'2, Kenji Tateda1,3, Ima Kosukegawa3 ${ }^{3}$, Satoshi Watanabe², \\ Toshihiko Yamashita ${ }^{3}$ \\ ${ }^{1}$ Department of Musculoskeletal Biomechanics and Surgical Development, Sapporo Medical University, \\ Sapporo, Japan \\ ${ }^{2}$ Department of Legal Medicine, Sapporo Medical University, Sapporo, Japan \\ ${ }^{3}$ Department of Orthopedic Surgery, Sapporo Medical University, Sapporo, Japan \\ Email: oka@sapmed.ac.jp
}

Received 6 May 2016; accepted 4 June 2016; published 7 June 2016

Copyright (C) 2016 by authors and Scientific Research Publishing Inc.

This work is licensed under the Creative Commons Attribution International License (CC BY). http://creativecommons.org/licenses/by/4.0/

(c) (i) Open Access

\begin{abstract}
Non-traumatic osteonecrosis of the femoral head (ONFH) is believed to be avascular necrosis. Certainly, a disruption of a supplying artery induces necrosis in the supplying artery-dominant region. However, when an abnormality such as cell apoptosis, or another reason, is developed in the supplying artery-dominant region, it induces a disruption of the supplying artery. Therefore, to prove that ONFH is avascular necrosis, it is necessary to demonstrate the disruption of the supplying artery prior to histological osteonecrosis development. Here we investigate histologically, using a rat model, whether disruption of the supplying artery occurs before the initial development of ONFH following corticosteroid treatment. Rats were given imiquimod and methylprednisolone, and were sacrificed 1, 2, 3, 7 or 14 days after the last injection. At the sacrifice, the rat was perfused with $20 \mathrm{ml}$ black Indian ink through the left ventricle. ONFH was observed in the Imiquimod + Methylprednisolone group at 7 and 14 days. The osteonecrotic area was not stained with perfused black Indian ink. However, the lateral portion of the femoral head near the superior retinacular artery was stained with the ink. In conclusion, the present study shows that the superior retinacular artery did not occlude before the initial development of ONFH histologically in rats.
\end{abstract}

\section{Keywords}

Osteonecrosis of the Femoral Head, Corticosteroid, Rat, Avascular 


\section{Introduction}

Corticosteroid-induced osteonecrosis of the femoral head (ONFH) is believed to be a multifactorial disease, and an avascular necrosis [1] [2]. Several reports demonstrated the disruption of the supplying artery as a cause of osteonecrosis in experimental animals and clinical reports [3] [4]. However, these reports merely present results obtained histologically or from diagnostic images after the initial development of osteonecrosis. Therefore, it still remains unclear whether corticosteroid treatment leads to a disruption of the supplying artery, and whether it induces ONFH.

We previously reported that corticosteroid treatment after an imiquimod (a toll-like receptor (TLR) 7 ligand) injection induces ONFH in Wistar ST rats [5]. The aim of the present study was to evaluate whether TLR7 stimulation by imiquimod and corticosteroid treatment leads to the disruption of the supplying artery before the development of ONFH histologically in rats.

\section{Materials and Methods}

\subsection{Animals}

All experiments were conducted in accordance with the guidelines of the Ministry of Sports, Culture, Science, and Technology of Japan, and followed protocols approved by the Animal Ethics Committee of Sapporo Medical University (\#12-084). Male Wistar ST rats (16 weeks old age, 300 - 350 g) were obtained from Sankyo Labo Service Co. Ltd. (Sapporo, Japan). All animals were housed in temperature- and humidity-controlled rooms with unlimited food and water and a 12-h light/dark cycle.

\subsection{Experimental Groups and Protocols}

Animals $(n=36)$ were treated as follows: the Control group $(n=6)$ were given saline $(1.0 \mathrm{ml} / \mathrm{kg})$ subcutaneously on Day 1 and saline $(1.0 \mathrm{ml} / \mathrm{kg})$ intramuscularly on Day 2 and sacrificed on Day 3; the Imiquimod + MPSL group ( $\mathrm{n}=30$ ) were given $30 \mathrm{mg} / \mathrm{kg}$ Imiquimod (Tokyo Chemical Industry, Tokyo, Japan) intramuscularly on Day 1 and 20 mg/kg methylprednisolone (Sigma, St Louis, MO, USA) intramuscularly on Day 2 as described previously [5]. Animals were sacrificed $1,2,3,7$ or 14 days ( $n=6$ each day) after the last injection. All injections were performed at 7:00 p.m. At the sacrifice, the rat was perfused with $20 \mathrm{ml}$ black Indian ink (KAIMEI \& Co., Ltd., Saitama, Japan) through the left ventricle, to act as an index of the blood flow in the femoral head. The femurs were harvested and fixed in a 10\% formalin-0.1 M phosphate buffer (pH 7.4).

\subsection{Histopathology}

The bone samples were decalcified with KalkitoxTM (Wako Pure Chemical Industries, Ltd. Osaka, Japan) and then neutralized with a 5\% sodium sulfate buffer. The tissues were then processed for non-staining and routine hematoxylin and eosin staining to assess the general architecture. Osteonecrosis was defined as the diffuse presence of empty lacunae or pyknotic nuclei in osteocytes within the bone trabeculae, accompanied by surrounding bone marrow cell necrosis [6]-[8].

\section{Results}

ONFH was observed in zero of 6 rats in the control group and in the Imiquimod + MPSL group at 1, 2 and 3 days, in 2 of 6 rats in the Imiquimod + MPSL group at 7 days, and in one of 6 rats in the Imiquimod + MPSL group at 14 days. All femoral heads showed staining with black Indian ink in the control group and in the Imiquimod + MPSL group at 1, 2 and 3 days in non-staining slides (Figure 1A, Figure 1C, Figure 1E, Figure 1G, respectively). Routinehematoxylin and eosin staining shows the normal trabeculae and hematopoietic and fat cells were observed in the control group and in the Imiquimod + MPSL group at 1, 2 and 3 days (Figure 1B, Figure 1D, Figure 1F, Figure 1H, respectively). However, the femoral head of one rat in the Imiquimod + MPSL group at 3 days showed only diffuse empty lacunae at bone trabeculae without bone marrow cell necrosis in the medullary space, and black Indian ink staining was weak in the central area of the femoral head with diffuse empty lacunae (Figure 2). In the Imiquimod + MPSL group at 7 days, rats with ONFH showed empty lacunae at the necrotic bone trabeculae, and an accumulation of cell debris in the medullary space in most areas of the femoral head (Figure 3B and Figure 3D), and the area of ONFH did not stain with black Indian ink (Figure 3A 

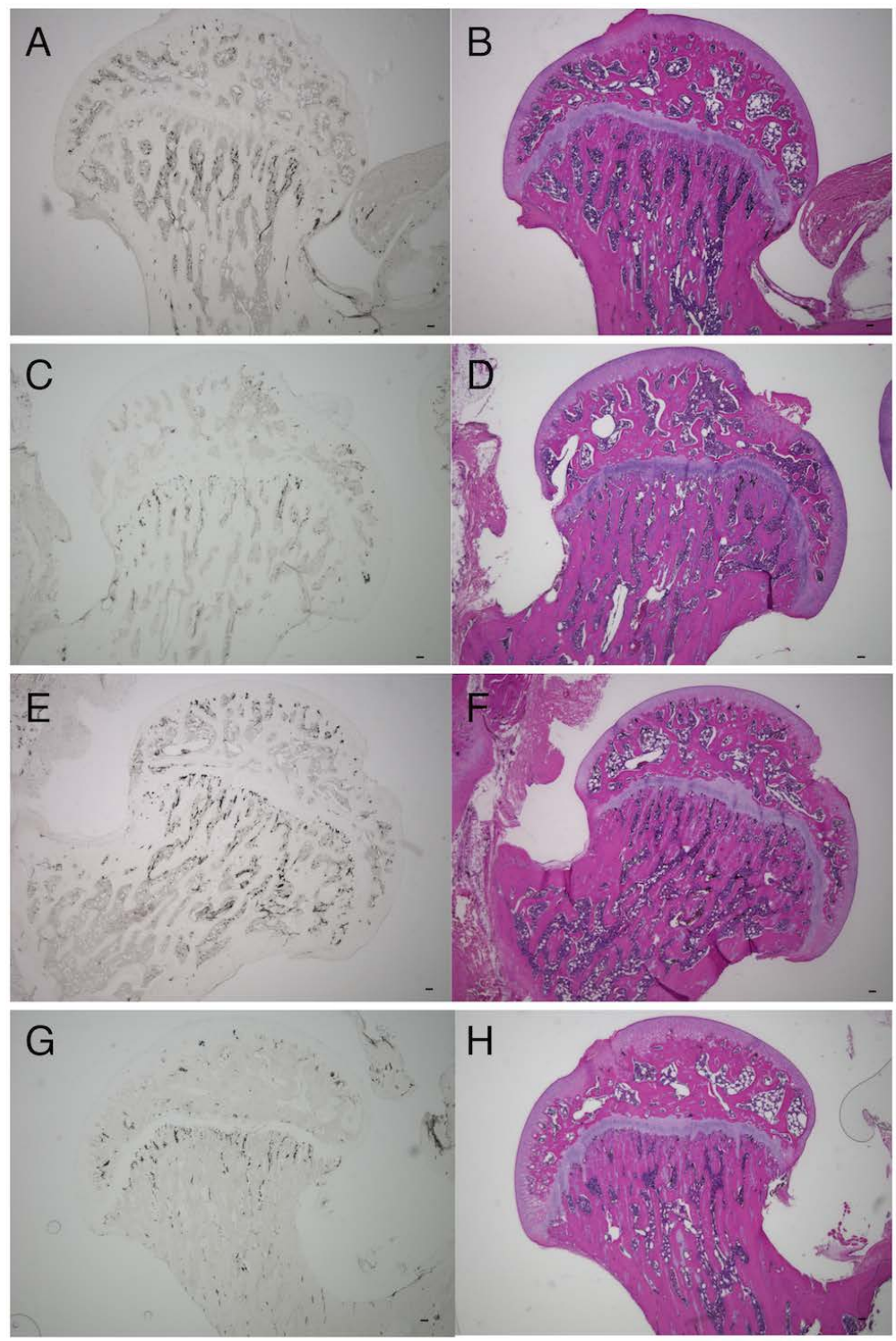

Figure 1. Hisological appearance of the femoral head. Panels show non-staining and hematoxylin and eosin stained images of the femoral head. Typical images for the Control (A, B), Imiquimod + MPSL 1 day (C, D), 2 days (E, F) and 3 days (G, H) groups are shown. All femoral heads showed staining with black Indian ink in non-staining slides (A, C, E, G). Routine hematoxylin and eosin staining shows the normal trabeculae and hematopoietic and fat cells (B, D, F, H). Scale bar: $100 \mu \mathrm{m}$.

and Figure 3C). However, the lateral portion of the femoral head near the superior retinacular artery stained with black Indian ink (Figure 3A and Figure 3E), and normal trabeculae and hematopoietic cells were observed with hematoxylin and eosin staining (Figure 3F). In the Imiquimod + MPSL group at 14 days, the rat with ONFH also showed similar histopathological findings to the Imiquimod + MPSL group at 7 days rats with ONFH (Figures 4A-C). Furthermore, formation of appositional bone around the necrotic bone trabeculae, as part of the repair process, and the deposition of fibrous and granulation tissue in the medullary space were observed at the lateral portion of the femoral head (Figure 4D).

\section{Discussion}

Osteonecrosis after high dose corticosteroid therapy often develops in the femoral head [9]. This may be due to 


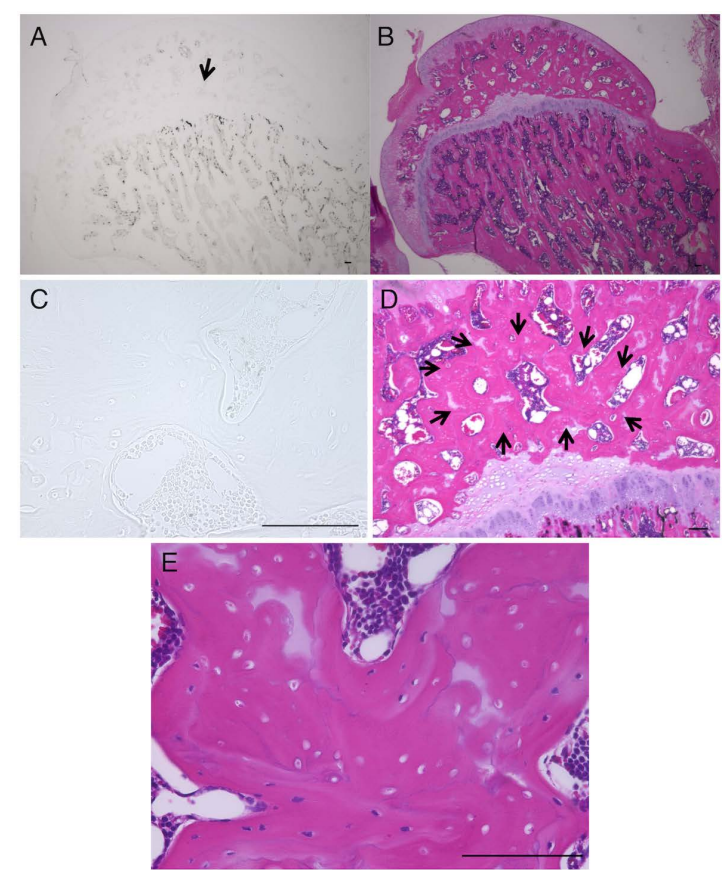

Figure 2. Hisological appearance of the femoral head in the Imiquimod+MPSL group of 3 days. Routine hematoxylin and eosin staining shows the normal-looking trabeculae and hematopoietic and fat cells (B, D). However, only diffuse empty lacunae at bone trabeculae were observed in the central area of the femoral head (D: black arrow, E). Black Indian ink staining was weak in the central area of the femoral head with diffuse empty lacunae (A, C). Scale bar: $100 \mu \mathrm{m}$.

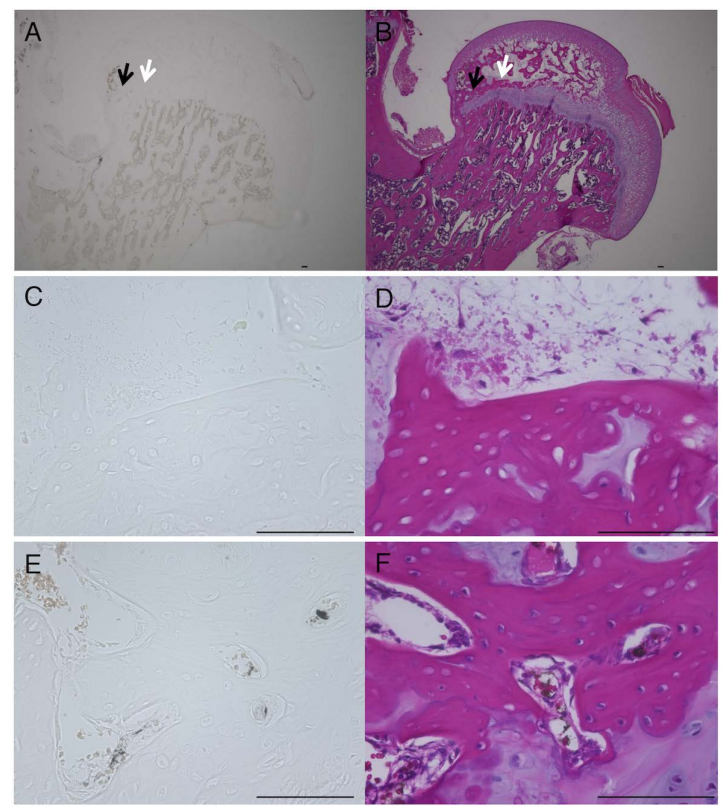

Figure 3. Hisological appearance of the femoral head in the Imiquimod+MPSL group of 7 days with ONFH. Diffuse empty lacunae at the necrotic bone trabeculae, and an accumulation of cell debris in the medullary space were observed in most areas of the femoral head with routine hematoxylin and eosin staining (B, D: white arrow), and the area of ONFH did not stain with black Indian ink (A, C: white arrow). The lateral portion of the femoral head bear the superior retinacular artery stained with black Indian ink (A, E: black arrow), and normal trabeculae and hematopoietic dells were observed with hematoxylin and eosin staining (F: black arrow). Scale bar: $100 \mu \mathrm{m}$. 

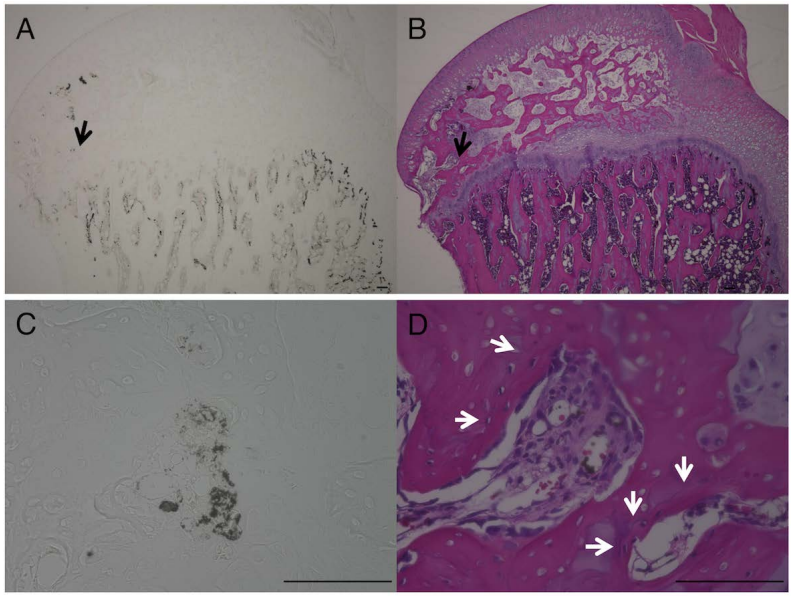

Figure 4. Hisological appearance of the femoral head in the Imiquimod+MPSL group of 14 days with ONFH. Diffuse empty lacunae at the necrotic bone trabeculae, and an accumulation of cell debris in the medullary space were observed in most areas of the femoral head with routine hematoxylin and eosin staining (B), and the area of ONFH did not stain with black Indian ink (A). The lateral portion of the femoral head bear the superior retinacular artery stained with black Indian ink (A, C: black arrow), and formation of appositional bone were observed with hematoxylin and eosin staining (B, D: black and white arrow). Scale bar: $100 \mu \mathrm{m}$.

the specific vascular anatomy of the femoral head [10]. Consequently, ONFH is believed to be avascular necrosis. Certainly, a disruption of a supplying artery induces necrosis in the supplying artery-dominant region. However, when an abnormality such as cell apoptosis, or another reason is developed in a supplying artery-dominant region, it induces a disruption of the supplying artery. Therefore, to prove that ONFH is avascular necrosis, it is necessary to demonstrate the disruption of the supplying artery prior to histological osteonecrosis development. It was reported that the superior retinacular arteries are important supplying arteries to the femoral head [10] [11]. In the present study, we observed that the lateral portion of femoral heads near the superior retinacular artery were stained with black Indian ink, and normal trabeculae and hematopoietic and fat cells were observed with or without formation of appositional bone in the osteonecrotic femoral head. This result indicates that the occlusion of the superior retinacular artery is not the cause of ONFH in rats. In addition, we observed that a femoral head of one rat in the Imiquimod + MPSL group at 3 days showed only diffuse empty lacunae at bone trabeculae, and that black Indian ink staining was weak in the central area of the femoral head with diffuse empty lacunae. Whether this change induces histological ONFH is not evident. It also still remains unclear whether osteocytes in bone trabeculae or bone marrow cells died earlier. Consequently, these results indicate that disruption of intraosseous micro vessels, or another causes such as osteocyte and bone marrow cells apoptosis, induces ONFH.

In conclusions, the present study shows that TLR7 stimulation and corticosteroid treatment did not lead to the disruption of the supplying artery before the initial development of ONFH histologically in rats. Thus, further analyses are necessary to prove that ONFH is avascular necrosis.

\section{Acknowledgements}

This work was supported in part by Grants-in-Aid for Young Scientists (B) (S.O., 25861330) from the Japan Society for the Promotion of Science.

\section{Conflict of Interest}

The authors have no conflicts of interest to declare.

\section{References}

[1] Chang, C.C., Greenspan, A. and Gershwin, M.E. (1993) Osteonecrosis: Current Perspectives on Pathogenesis and Treatment. Seminars in Arthritis and Rheumatism, 23, 47-69. http://dx.doi.org/10.1016/S0049-0172(05)80026-5 
[2] Mont, M.A., Cherian, J.J., Sierra, R.J., Jones, L.C. and Lieberman, J.R. (2015) Nontraumatic Osteonecrosis of the Femoral Head: Where Do We Stand Today? A Ten-Year Update. The Journal of Bone \& Joint Surgery American, 97, 1604-1627. http://dx.doi.org/10.2106/JBJS.O.00071

[3] Atsumi, T. and Kuroki, Y. (1992) Role of Impairment of Blood Supply of the Femoral Head in the Pathogenesis of Idiopathic Osteonecrosis. Clinical Orthopaedics and Related Research, 277, 22-30.

[4] Janke, L.J., Liu, C., Vogel, P., Kawedia, J., Boyd, K.L., Funk, A.J. and Relling, M.V. (2013) Primary Epiphyseal Arteriopathy in a Mouse Model of Steroid-Induced Osteonecrosis. The American Journal of Pathology, 183, 19-25. http://dx.doi.org/10.1016/j.ajpath.2013.03.004

[5] Okazaki, S., Nagoya, S., Matsumoto, H., Mizuo, K., Sasaki, M., Watanabe, S. and Inoue, H. (2015) Development of Non-Traumatic Osteonecrosis of the Femoral Head Requires Toll-Like Receptor 7 and 9 Stimulations and Is Boosted by Repression on Nuclear Factor Kappa B in Rats. Laboratory Investigation, 95, 92-99. http://dx.doi.org/10.1038/labinvest.2014.134

[6] Yamamoto, T., Hirano, K., Tsutsui, H., Sugioka, Y. and Sueishi, K. (1995) Corticosteroid Enhances the Experimental Induction of Osteonecrosis in Rabbits with Shwartzman Reaction. Clinical Orthopaedics and Related Research, 316, 235-243. http://dx.doi.org/10.1097/00003086-199507000-00033

[7] Okazaki, S., Nishitani, Y., Nagoya, S., Kaya, M., Yamashita, T. and Matsumoto, H. (2009) Femoral Head Osteonecrosis Can Be Caused by Disruption of the Systemic Immune Response via the Toll-Like Receptor 4 Signalling Pathway. Rheumatology, 48, 227-232. http://dx.doi.org/10.1093/rheumatology/ken462

[8] Okazaki, S., Nagoya, S., Tateda, K., Katada, R., Mizuo, K., Watanabe, S. and Matsumoto, H. (2013) Experimental Rat Model for Alcohol-Induced Osteonecrosis of the Femoral Head. International Journal of Experimental Pathology, 94, 312-319. http://dx.doi.org/10.1111/iep.12035

[9] Sugioka, Y. (1978) Transtrochanteric Anterior Rotational Osteotomy of the Femoral Head in the Treatment of Osteonecrosis Affecting the Hip: A New Osteotomy Operation. Clinical Orthopaedics and Related Research, 130, 191-201. http://dx.doi.org/10.1097/00003086-197801000-00019

[10] Trueta, J. and Harrison, M.H. (1953) The Normal Vascular Anatomy of the Femoral Head in Adult Man. The Journal of Bone and Joint Surgery. British Volume, 35-B, 442-461.

[11] Theron, J. (1977) Superselective Angiography of the Hip. Technique, Normal Features, and Early Results in Idiopathic Necrosis of the Femoral Head. Radiology, 124, 649-657. http://dx.doi.org/10.1148/124.3.649 